\title{
WEIGHTED CONTINUITY OF MULTILINEAR MARCINKIEWICZ OPERATORS FOR THE EXTREME CASES OF $p$
}

\section{LIU LANZHE}

ABSTRACT. In this paper, we prove the weighted continuity of multilinear Marcinkiewicz operators for the extreme cases of $p$.

\section{Introduction and results}

Suppose that $S^{n-1}$ is the unit sphere of $R^{n}(n \geq 2)$ equipped with normalized Lebesgue measure $d \sigma=d \sigma\left(x^{\prime}\right)$. Let $\Omega$ be homogeneous of degree zero and satisfy the following two conditions:

(i) $\Omega(x)$ is continuous on $S^{n-1}$ and satisfies the $\operatorname{Lip}_{\gamma}$ condition on $S^{n-1}(0<\gamma \leq 1)$, i.e.

$$
\left|\Omega\left(x^{\prime}\right)-\Omega\left(y^{\prime}\right)\right| \leq M\left|x^{\prime}-y^{\prime}\right|^{\gamma}, \quad x^{\prime}, y^{\prime} \in S^{n-1} ;
$$

(ii) $\int_{S^{n-1}} \Omega\left(x^{\prime}\right) d x^{\prime}=0$.

Let $m$ be a positive integer and $A$ be a function on $R^{n}$. We denote that $\Gamma(x)=\left\{(y, t) \in R_{+}^{n+1}:|x-y|<t\right\}$ and the characteristic of $\Gamma(x)$ by $\chi_{\Gamma(x)}$. The multilinear Marcinkiewicz operator is defined by

$$
\mu_{S}^{A}(f)(x)=\left[\iint_{\Gamma(x)}\left|F_{t}^{A}(f)(x, y)\right|^{2} \frac{d y d t}{t^{n+3}}\right]^{1 / 2},
$$

where

$$
F_{t}^{A}(f)(x, y)=\int_{|y-z| \leq t} \frac{\Omega(y-z)}{|y-z|^{n-1}} \frac{R_{m+1}(A ; x, z)}{|x-z|^{m}} f(z) d z
$$

Received March 18, 2003.

2000 Mathematics Subject Classification: 42B20, 42B25.

Key words and phrases: Marcinkiewicz integral operator, multilinear operators, BMO space, hardy space.

Supported by the NNSF (Grant: 10271071). 
and

$$
R_{m+1}(A ; x, y)=A(x)-\sum_{|\alpha| \leq m} \frac{1}{\alpha !} D^{\alpha} A(y)(x-y)^{\alpha} .
$$

We denote that

$$
F_{t}(f)(y)=\int_{|y-z| \leq t} \frac{\Omega(y-z)}{|y-z|^{n-1}} f(z) d z .
$$

We also define that

$$
\mu_{S}(f)(x)=\left(\iint_{\Gamma(x)}\left|F_{t}(f)(y)\right|^{2} \frac{d y d t}{t^{n+3}}\right)^{1 / 2}
$$

which is the Marcinkiewicz integral operator (see [10]).

Let $H$ be the Hilbert space

$$
H=\left\{h:\|h\|=\left(\iint_{R_{+}^{n+1}}|h(t)|^{2} d y d t / t^{n+3}\right)^{1 / 2}<\infty\right\},
$$

then for each fixed $x \in R^{n}, F_{t}^{A}(f)(x, y)$ may be viewed as a mapping from $(0,+\infty)$ to $H$, and it is clear that

$$
\begin{gathered}
\mu_{S}^{A}(f)(x)=\left\|\chi_{\Gamma(x)} F_{t}^{A}(f)(x, y)\right\| \\
\mu_{S}(f)(x)=\left\|\chi_{\Gamma(x)} F_{t}(f)(y)\right\| .
\end{gathered}
$$

We also consider the variant of $\mu_{S}^{A}$, which is defined by

$$
\tilde{\mu}_{S}^{A}(f)(x)=\left(\iint_{\Gamma(x)}\left|\tilde{F}_{t}^{A}(f)(x, y)\right|^{2} \frac{d y d t}{t^{n+3}}\right)^{1 / 2}
$$

where

$$
\tilde{F}_{t}^{A}(f)(x, y)=\int_{|y-z| \leq t} \frac{\Omega(y-z)}{|y-z|^{n-1}} \frac{Q_{m+1}(A ; x, z)}{|x-z|^{m}} f(z) d z
$$

and

$$
Q_{m+1}(A ; x, z)=R_{m}(A ; x, z)-\sum_{|\alpha|=m} \frac{1}{\alpha !} D^{\alpha} A(x)(x-z)^{\alpha} .
$$

Note that when $m=0, \mu_{S}^{A}$ is just the commutator of Marcinkiewicz integral operator (see [15], [18]). It is well known that multilinear operators, as the extension of commutators, are of great interest in harmonic analysis and have been widely studied by many authors (see 
$[3-6],[8],[9],[13],[14])$. In [12], the endpoint boundedness properties of the commutators generated by the Calderon-Zygmund operator and BMO functions are obtained. The main purpose of this paper is to discuss the weighted continuity properties of the multilinear Marcinkiewicz operators for the extreme cases of $p$. Throughout this paper, $B$ will denote a ball of $R^{n}$. For a ball $B$ and any locally integral function $f$ on $R^{n}$, we denote that $f(B)=\int_{B} f(x) d x$, $f_{B}=|B|^{-1} \int_{B} f(x) d x$ and $f^{\#}(x)=\sup _{x \in B}|B|^{-1} \int_{B}\left|f(y)-f_{B}\right| d y$. Moreover, for a weight functions $w \in A_{1}$ (see [11]), $f$ is said to belong to $B M O(w)$ if $f^{\#} \in L^{\infty}(w)$ and define $\|f\|_{B M O(w)}=\left\|f^{\#}\right\|_{L^{\infty}(w)}$, if $w=1$, we denote that $B M O\left(R^{n}\right)=B M O(w)$. Also, we give the concepts of atom and weighted $H^{1}$ space. A function $a$ is called a $H^{1}(w)$ atom if there exists a ball $B$ such that $a$ is supported on $B,\|a\|_{L^{\infty}(w)} \leq w(B)^{-1}$ and $\int_{R^{n}} a(x) d x=0$. It is well known that, for $w \in A_{1}$, the weighted Hardy space $H^{1}(w)$ has the atomic decomposition characterization(see $[1])$.

We shall prove the following theorems in Section 3.

Theorem 1. Let $D^{\alpha} A \in B M O\left(R^{n}\right)$ for $|\alpha|=m$ and $w \in A_{1}$. Then $\mu_{S}^{A}$ maps $L^{\infty}(w)$ continuously into $B M O(w)$.

THEOREM 2. Let $D^{\alpha} A \in B M O\left(R^{n}\right)$ for $|\alpha|=m$ and $w \in A_{1}$. Then $\tilde{\mu}_{S}^{A}$ maps $H^{1}(w)$ continuously into $L^{1}(w)$.

Theorem 3. Let $D^{\alpha} A \in B M O\left(R^{n}\right)$ for $|\alpha|=m$ and $w \in A_{1}$. Then $\mu_{S}^{A}$ maps $H^{1}(w)$ continuously into weak $L^{1}(w)$.

Theorem 4. Let $D^{\alpha} A \in B M O\left(R^{n}\right)$ for $|\alpha|=m$ and $w \in A_{1}$.

(i) If for any $H^{1}(w)$-atom a supported on certain cube $Q$ and $u \in$ $3 Q \backslash 2 Q$, there is

$$
\begin{aligned}
& \int_{(4 Q)^{c}} \| \chi_{\Gamma(x)} \sum_{|\alpha|=m} \frac{1}{\alpha !} \frac{(x-u)^{\alpha}}{|x-u|^{m}} \frac{\Omega(y-u)}{|y-u|^{n-1}} \chi_{\Gamma(y)}(u, t) \\
& \quad \times \int_{Q} D^{\alpha} A(z) a(z) d z \| w(x) d x \\
& \leq C,
\end{aligned}
$$

then $\mu_{S}^{A}$ is bounded from $H^{1}(w)$ to $L^{1}(w)$; 
(ii) If for any cube $Q$ and $u \in 3 Q \backslash 2 Q$, there is

$$
\begin{aligned}
& \frac{1}{w(Q)} \int_{Q} \| \chi_{\Gamma(x)} \sum_{|\alpha|=m} \frac{1}{\alpha !}\left(D^{\alpha} A(x)-\left(D^{\alpha} A\right)_{Q}\right) \\
& \quad \times \int_{(4 Q)^{c}} \frac{(u-z)^{\alpha}}{|u-z|^{m}} \frac{\Omega(y-z) \chi_{\Gamma(y)}(z, t)}{|y-z|^{n-1}} f(z) d z \| w(x) d x \\
& \leq C\|f\|_{L^{\infty}(w)},
\end{aligned}
$$

then $\tilde{\mu}_{S}^{A}$ is bounded from $L^{\infty}(w)$ to $B M O(w)$.

\section{Some lemmas}

We begin with some preliminary lemmas.

LEMmA 1. (see [6]) Let $A$ be a function on $R^{n}$ and $D^{\alpha} A \in L^{q}\left(R^{n}\right)$ for $|\alpha|=m$ and some $q>n$. Then

$$
\left|R_{m}(A ; x, y)\right| \leq C|x-y|^{m} \sum_{|\alpha|=m}\left(\frac{1}{|\tilde{B}(x, y)|} \int_{\tilde{B}(x, y)}\left|D^{\alpha} A(z)\right|^{q} d z\right)^{1 / q}
$$

where $\tilde{B}(x, y)$ is the ball centered at $x$ and having radius $5 \sqrt{n}|x-y|$.

LeMma 2. Let $w \in A_{1}, 1<p<\infty, 1<r \leq \infty, 1 / q=1 / p+1 / r$ and $D^{\alpha} A \in B M O\left(R^{n}\right)$ for $|\alpha|=m$. Then $\mu_{S}^{A}$ is bound from $L^{p}(w)$ to $L^{q}(w)$, that is

$$
\left\|\mu_{S}^{A}(f)\right\|_{L^{q}(w)} \leq C \sum_{|\alpha|=m}\left\|D^{\alpha} A\right\|_{B M O}\|f\|_{L^{p}(w)} .
$$

Proof. Note that $|x-z| \leq 2 t,|y-z| \geq|x-z|-t \geq|x-z|-3 t$ when $|x-y| \leq t,|y-z| \leq t$. By Minkowski inequality, we have

$$
\begin{aligned}
\mu_{S}^{A}(f)(x) \leq & \int_{R^{n}}\left[\iint_{|x-y| \leq t}\left(\frac{|\Omega(y-z)|\left|R_{m+1}(A ; x, z)\right||f(z)|}{|y-z|^{n-1}|x-z|^{m}}\right)^{2}\right. \\
& \left.\times \chi_{\Gamma(z)}(y, t) \frac{d y d t}{t^{n+3}}\right]^{1 / 2} d z \\
\leq & C \int_{R^{n}} \frac{\left|R_{m+1}(A ; x, z)\right||f(z)|}{|x-z|^{m}}
\end{aligned}
$$




$$
\begin{aligned}
& \times\left[\iint_{|x-y| \leq t} \frac{\chi_{\Gamma(z)}(y, t) t^{-n-3}}{(|x-z|-3 t)^{2 n-2}} d y d t\right]^{1 / 2} d z \\
\leq & C \int_{R_{n}} \frac{\left|R_{m+1}(A ; x, z)\right||f(z)|}{|x-z|^{m+3 / 2}} \\
& \times\left[\int_{|x-z| / 2}^{\infty} \frac{d t}{(|x-z|-3 t)^{2 n-2}}\right]^{1 / 2} d z \\
\leq & C \int_{R_{n}} \frac{\left|R_{m+1}(A ; x, z)\right|}{|x-z|^{m+n}}|f(z)| d z
\end{aligned}
$$

thus, the lemma follows from [8], [9].

\section{Proofs of theorems}

ProOF OF THEOREM 1. It is only to prove that there exists a constant $C_{B}$ such that

$$
\frac{1}{w(B)} \int_{B}\left|\mu_{S}^{A}(f)(x)-C_{B}\right| w(x) d x \leq C\|f\|_{L^{\infty}(w)}
$$

holds for any ball $B$. Fix a ball $B=B\left(x_{0}, l\right)$. Let $\tilde{B}=5 \sqrt{n} B$ and $\tilde{A}(x)=A(x)-\sum_{|\alpha|=m} \frac{1}{\alpha !}\left(D^{\alpha} A\right)_{\tilde{B}} x^{\alpha}$, then $R_{m}(A ; x, y)=R_{m}(\tilde{A} ; x, y)$ and $D^{\alpha} \tilde{A}=D^{\alpha} A-\left(D^{\alpha} A\right)_{\tilde{B}}$ for $|\alpha|=m$. We write, for $f_{1}=f \chi_{\tilde{B}}$ and $f_{2}=f \chi_{R^{n} \backslash \tilde{B}}$

$$
F_{t}^{A}(f)(x)=F_{t}^{A}\left(f_{1}\right)(x)+F_{t}^{A}\left(f_{2}\right)(x)
$$

then

$$
\begin{aligned}
& \frac{1}{w(B)} \int_{B}\left|\mu_{S}^{A}(f)(x)-\mu_{S}^{A}\left(f_{2}\right)\left(x_{0}\right)\right| w(x) d x \\
= & \frac{1}{w(B)} \int_{B}\left\|\chi_{\Gamma(x)} F_{t}^{A}(f)(x, y)\right\|-\left\|\chi_{\Gamma(x)} F_{t}^{A}\left(f_{2}\right)\left(x_{0}, y\right)\right\| \| w(x) d x \\
\leq & \frac{1}{w(B)} \int_{B} \mu_{S}^{A}\left(f_{1}\right)(x) w(x) d x \\
& +\frac{1}{w(B)} \int_{B}\left\|\chi_{\Gamma(x)} F_{t}^{A}\left(f_{2}\right)(x, y)-\chi_{\Gamma(x)} F_{t}^{A}\left(f_{2}\right)\left(x_{0}, y\right)\right\| w(x) d x \\
:= & I+I I .
\end{aligned}
$$


Now, let us estimate $I$ and $I I$. First, by the $L^{\infty}$ boundedness of $\mu_{S}^{A}$ (Lemma 2), we gain

$$
I \leq\left\|\mu_{S}^{A}\left(f_{1}\right)\right\|_{L^{\infty}(w)} \leq C\|f\|_{L^{\infty}(w)} .
$$

To estimate $I I$, we write

$$
\begin{aligned}
& \chi_{\Gamma(x)} F_{t}^{A}\left(f_{2}\right)(x, y)-\chi_{\Gamma(x)} F_{t}^{A}\left(f_{2}\right)\left(x_{0}, y\right) \\
= & \int_{|y-z| \leq t}\left[\frac{1}{|x-z|^{m}}-\frac{1}{\left|x_{0}-z\right|^{m}}\right] \frac{\chi_{\Gamma(x)} \Omega(y-z) R_{m}(\tilde{A} ; x, z) f_{2}(z)}{|y-z|^{n-1}} d z \\
& +\int_{|y-z| \leq t} \frac{\chi_{\Gamma(x)} \Omega(y-z) f_{2}(z)}{|y-z|^{n-1}\left|x_{0}-z\right|^{m}}\left[R_{m}(\tilde{A} ; x, z)-R_{m}\left(\tilde{A} ; x_{0}, z\right)\right] d z \\
& +\int_{|y-z| \leq t}\left(\chi_{\Gamma(x)}-\chi_{\Gamma\left(x_{0}\right)}\right) \frac{\Omega(y-z) R_{m}\left(\tilde{A} ; x_{0}, z\right) f_{2}(z)}{|y-z|^{n-1}\left|x_{0}-z\right|^{m}} d z \\
& -\sum_{|\alpha|=m} \frac{1}{\alpha !} \int_{|y-z| \leq t}\left[\frac{\chi_{\Gamma(x)}(x-z)^{\alpha}}{|x-z|^{m}}-\frac{\chi_{\Gamma\left(x_{0}\right)}\left(x_{0}-z\right)^{\alpha}}{\left|x_{0}-z\right|^{m}}\right] \\
& \times \frac{\Omega(y-z) D^{\alpha} \tilde{A}(z) f_{2}(z)}{|y-z|^{n-1}} d z \\
:= & I I_{1}^{t}(x)+I I_{2}^{t}(x)+I I_{3}^{t}(x)+I I_{4}^{t}(x) .
\end{aligned}
$$

Note that $|x-z| \sim\left|x_{0}-z\right|$ for $x \in \tilde{B}$ and $z \in R^{n} \backslash \tilde{B}$, and by the similar method to the proof of Lemma 2 and by Lemma 1, we have

$$
\begin{aligned}
& \frac{1}{w(B)} \int_{B}\left\|I I_{1}^{t}(x)\right\| w(x) d x \\
\leq & \frac{C}{w(B)} \int_{B}\left(\int_{R^{n} \backslash \tilde{B}} \frac{\left|x-x_{0}\right||f(z)|}{\left.|x-z|^{n+m+1}\left|R_{m}(\tilde{A} ; x, z)\right| d z\right) w(x) d x}\right. \\
\leq & \frac{C}{w(B)} \int_{B}\left(\sum_{k=0}^{\infty} \int_{2^{k+1} \tilde{B} \backslash 2^{k} \tilde{B}} \frac{\left|x-x_{0}\right||f(z)|}{\left.|x-z|^{n+m+1}\left|R_{m}(\tilde{A} ; x, z)\right| d z\right) w(x) d x}\right. \\
\leq & C \sum_{k=1}^{\infty} \frac{k l\left(2^{k} l\right)^{m}}{\left(2^{k} l\right)^{n+m+1}} \sum_{|\alpha|=m}\left\|D^{\alpha} A\right\|_{B M O}\left(\int_{2^{k+1} \tilde{B}}|f(z)| d z\right) \\
\leq & C \sum_{|\alpha|=m}\left\|D^{\alpha} A\right\|_{B M O}\|f\|_{L^{\infty}(w)} \sum_{k=1}^{\infty} k 2^{-k} \\
\leq & C \sum_{|\alpha|=m}\left\|D^{\alpha} A\right\|_{B M O}\|f\|_{L^{\infty}(w)}
\end{aligned}
$$


For $I I_{2}^{t}(x)$, by the formula (see [6]):

$$
\begin{aligned}
& R_{m}(\tilde{A} ; x, z)-R_{m}\left(\tilde{A} ; x_{0}, z\right) \\
= & R_{m}\left(\tilde{A} ; x, x_{0}\right) \\
& +\sum_{0<|\beta|<m} \frac{1}{\beta !} R_{m-|\beta|}\left(D^{\beta} \tilde{A} ; x_{0}, z\right)\left(x-x_{0}\right)^{\beta}
\end{aligned}
$$

and Lemma 1, we get

$$
\begin{aligned}
& \left|R_{m}(\tilde{A} ; x, z)-R_{m}\left(\tilde{A} ; x_{0}, z\right)\right| \\
\leq & C \sum_{|\alpha|=m}|| D^{\alpha} A \|_{B M O}\left(\left|x-x_{0}\right|^{m}\right. \\
& \left.+\sum_{0<|\beta|<m}\left|x_{0}-z\right|^{m-|\beta|}\left|x-x_{0}\right|^{|\beta|}\right),
\end{aligned}
$$

thus, for $x \in B$,

$$
\begin{aligned}
& \left\|I I_{2}^{t}(x)\right\| \\
\leq & C \int_{R^{n}} \frac{\left|f_{2}(z)\right|}{|x-z|^{m+n}}\left|R_{m}(\tilde{A} ; x, z)-R_{m}\left(\tilde{A} ; x_{0}, z\right)\right| d z \\
\leq & C \sum_{|\alpha|=m}\left\|D^{\alpha} A\right\|_{B M O} \\
& \times \int_{R^{n}} \frac{\left|x-x_{0}\right|^{m}+\sum_{0<|\beta|<m}\left|x_{0}-z\right|^{m-|\beta|}\left|x-x_{0}\right|^{|\beta|}}{\left|x_{0}-z\right|^{m+n}}\left|f_{2}(z)\right| d z \\
\leq & C \sum_{|\alpha|=m}\left\|D^{\alpha} A\right\|_{B M O} \sum_{k=0}^{\infty} \frac{k l^{m}}{\left(2^{k} l\right)^{m+n}} \int_{2^{k+1} \tilde{B}}|f(z)| d z \\
\leq & C \sum_{|\alpha|=m}\left\|D^{\alpha} A\right\|_{B M O}\|f\|_{L^{\infty}(w)} \sum_{k=1}^{\infty} k 2^{-k m} \\
\leq & C \sum_{|\alpha|=m}\left\|D^{\alpha} A\right\|_{B M O}\|f\|_{L^{\infty}(w)} ;
\end{aligned}
$$

For $I I_{3}^{t}(x)$, note that $|x+y-z| \sim\left|x_{0}+y-z\right|$ for $x \in \tilde{B}$ and $z \in R^{n} \backslash \tilde{B}$, we obtain from the similar method to the estimate of $I I_{1}$,

$$
\left\|I I_{3}^{t}(x)\right\| \leq C \int_{R^{n}}\left(\int _ { R _ { + } ^ { n + 1 } } \left[\frac{\left|f_{2}(z)\right||\Omega(y-z)| \chi_{\Gamma(z)}(y, t)\left|R_{m}\left(\tilde{A} ; x_{0}, z\right)\right|}{|y-z|^{n-1}\left|x_{0}-z\right|^{m}}\right.\right.
$$




$$
\begin{aligned}
& \left.\left.\times\left(\chi_{\Gamma(x)}(y, t)-\chi_{\Gamma\left(x_{0}\right)}(y, t)\right)\right]^{2} \frac{d y d t}{t^{n+3}}\right)^{1 / 2} d z \\
& \leq C \int_{R^{n}} \frac{\left|f_{2}(z)\right|\left|R_{m}\left(\tilde{A} ; x_{0}, z\right)\right|}{\left|x_{0}-z\right|^{m}} \mid \iint_{|x-y| \leq t} \frac{t^{-n-3} \chi_{\Gamma(z)}(y, t)}{|y-z|^{2 n-2}} d y d t \\
& -\left.\iint_{\left|x_{0}-y\right| \leq t} \frac{t^{-n-3} \chi_{\Gamma(z)}(y, t)}{|y-z|^{2 n-2}} d y d t\right|^{1 / 2} d z \\
& \leq C \int_{R^{n}} \frac{\left|f_{2}(z)\right|\left|R_{m}\left(\tilde{A} ; x_{0}, z\right)\right|}{\left|x_{0}-z\right|^{m}}\left(\iint_{|y| \leq t,|x+y-z| \leq t} \mid \frac{1}{|x+y-z|^{2 n-2}}\right. \\
& -\frac{1}{\left|x_{0}+y-z\right|^{2 n-2}}\left(\frac{d y d t}{t^{n+3}}\right)^{1 / 2} d z \\
& \leq C \int_{R^{n}} \frac{\left|f_{2}(z)\right|\left|R_{m}\left(\tilde{A} ; x_{0}, z\right)\right|}{\left|x_{0}-z\right|^{m}} \\
& \times\left(\iint_{|y| \leq t,|x+y-z| \leq t} \frac{\left|x-x_{0}\right|}{|x+y-z|^{2 n+2}} t^{-n} d y d t\right)^{1 / 2} d z \\
& \leq C \int_{R^{n}} \frac{\left|f_{2}(z)\right|\left|x-x_{0}\right|^{1 / 2}\left|R_{m}\left(\tilde{A} ; x_{0}, z\right)\right|}{\left|x_{0}-z\right|^{m+n+1 / 2}} d z \\
& \leq C \sum_{k=0}^{\infty} \frac{k l^{1 / 2}\left(2^{k} l\right)^{m}}{\left(2^{k} l\right)^{n+m+1 / 2}} \sum_{|\alpha|=m}\left\|D^{\alpha} A\right\|_{B M O}\left(\int_{2^{k+1} \tilde{B}}|f(z)| d z\right) \\
& \leq C \sum_{|\alpha|=m}\left\|D^{\alpha} A\right\|_{B M O}\|f\|_{L^{\infty}(w)} \sum_{k=0}^{\infty} k 2^{-k / 2} \\
& \leq C \sum_{|\alpha|=m}\left\|D^{\alpha} A\right\|_{B M O}\|f\|_{L^{\infty}(w)}
\end{aligned}
$$

For $I I_{4}^{t}(x)$, by the similar method to the estimates of $I I_{1}^{t}(x)$ and $I I_{3}^{t}(x)$, we have

$$
\begin{aligned}
|| I I_{4}^{t}(x) \| \leq & C \int_{R^{n} \backslash \tilde{B}}\left[\frac{\left|x-x_{0}\right|}{|x-z|^{n+1}}+\frac{\left|x-x_{0}\right|^{1 / 2}}{|x-z|^{n+1 / 2}}\right] \\
& \times \sum_{|\alpha|=m}\left|D^{\alpha} \tilde{A}(z)\right||f(z)| d z
\end{aligned}
$$




$$
\begin{aligned}
& \leq C \sum_{|\alpha|=m}\left\|D^{\alpha} A\right\|_{B M O}\|f\|_{L^{\infty}(w)} \sum_{k=0}^{\infty} k\left(2^{-k}+2^{-k / 2}\right) \\
& \leq C \sum_{|\alpha|=m}\left\|D^{\alpha} A\right\|_{B M O}\|f\|_{L^{\infty}(w)} .
\end{aligned}
$$

Combining these estimates, we completes the proof of Theorem 1.

Proof of Theorem 2. It suffices to show that there exists a constant $C>0$ such that for every $H^{1}$-atom $a$ (that is that $a$ satisfies: $\operatorname{supp} a \subset B=B\left(x_{0}, r\right),\|a\|_{L^{\infty}(w)} \leq w(B)^{-1}$ and $\int_{R^{n}} a(y) d y=0$ (see $[1]))$, the following holds:

$$
\left\|\tilde{\mu}_{S}^{A}(a)\right\|_{L^{1}(w)} \leq C
$$

We write

$$
\begin{aligned}
\int_{R^{n}} \tilde{\mu}_{S}^{A}(a)(x) w(x) d x & =\left[\int_{\left|x-x_{0}\right| \leq 2 r}+\int_{\left|x-x_{0}\right|>2 r}\right] \tilde{\mu}_{S}^{A}(a)(x) w(x) d x \\
:=J+J J &
\end{aligned}
$$

For $J$, by the following equality

$$
Q_{m+1}(A ; x, y)=R_{m+1}(A ; x, y)-\sum_{|\alpha|=m} \frac{1}{\alpha !}(x-y)^{\alpha}\left(D^{\alpha} A(x)-D^{\alpha} A(y)\right)
$$

we have, by the similar method to the proof of Lemma 2,

$$
\tilde{\mu}_{S}^{A}(a)(x) \leq \mu_{S}^{A}(a)(x)+C \sum_{|\alpha|=m} \int_{R^{n}} \frac{\left|D^{\alpha} A(x)-D^{\alpha} A(y)\right|}{|x-y|^{n}}|a(y)| d y
$$

thus, $\tilde{\mu}_{\delta}^{A}$ is $L^{\infty}$-bounded by Lemma 2 and [2]. We see that

$$
J \leq C\left\|\tilde{\mu}_{S}^{A}(a)\right\|_{L^{\infty}(w)} w(2 B) \leq C\|a\|_{L^{\infty}(w)} w(B) \leq C .
$$

To obtain the estimate of $J J$, we denote that

$$
\tilde{A}(x)=A(x)-\sum_{|\alpha|=m} \frac{1}{\alpha !}\left(D^{\alpha} A\right)_{2 B} x^{\alpha}
$$

then $Q_{m}(A ; x, y)=Q_{m}(\tilde{A} ; x, y)$. We write, by the vanishing moment of $a$ and $Q_{m+1}(A ; x, y)=R_{m}(A ; x, y)-\sum_{|\alpha|=m} \frac{1}{\alpha !}(x-y)^{\alpha} D^{\alpha} A(x)$, for 
$x \in(2 B)^{c}$,

$$
\begin{aligned}
\tilde{F}_{t}^{A}(a)(x, y)= & \int_{|y-z| \leq t} \frac{\Omega(y-z) R_{m}(\tilde{A} ; x, z)}{|y-z|^{n-1}|x-z|^{m}} a(z) d z \\
& -\sum_{|\alpha|=m} \frac{1}{\alpha !} \int_{|y-z| \leq t} \frac{\Omega(y-z) D^{\alpha} \tilde{A}(x)(x-z)^{\alpha}}{|y-z|^{n-1}|x-z|^{m}} a(z) d z \\
= & \int_{R^{n}}\left[\frac{\chi_{\Gamma(y)}(z, t) \Omega(y-z) R_{m}(\tilde{A} ; x, z)}{|y-z|^{n-1}|x-z|^{m}}\right. \\
& \left.-\frac{\chi_{\Gamma(y)}\left(x_{0}, t\right) \Omega\left(y-x_{0}\right) R_{m}\left(\tilde{A} ; x, x_{0}\right)}{\left|y-x_{0}\right|^{n-1}\left|x-x_{0}\right|^{m}}\right] a(z) d z \\
& -\sum_{|\alpha|=m} \frac{1}{\alpha !} \int_{R^{n}}\left[\frac{\chi_{\Gamma(y)}(z, t) \Omega(y-z)(x-z)^{\alpha}}{|y-z|^{n-1}|x-z|^{m}}\right. \\
& \left.-\frac{\chi_{\Gamma(y)}\left(x_{0}, t\right) \Omega\left(y-x_{0}\right)\left(x-x_{0}\right)^{\alpha}}{\left|y-x_{0}\right|^{n-1}\left|x-x_{0}\right|^{m}}\right] D^{\alpha} \tilde{A}(x) a(z) d z
\end{aligned}
$$

thus, by the similar method to the proof of $I I$ in Theorem 1, we obtain

$$
\begin{aligned}
\left\|\tilde{F}_{t}^{A}(a)(x, y)\right\| \leq & C \frac{|B|^{1+1 / n}}{w(B)}\left(\sum_{|\alpha|=m}|| D^{\alpha} A \|_{B M O}\left|x-x_{0}\right|^{-n-1}\right. \\
& \left.+\left|x-x_{0}\right|^{-n-1}\left|D^{\alpha} \tilde{A}(x)\right|\right)
\end{aligned}
$$

note that if $w \in A_{1}$, then $\frac{w\left(B_{2}\right)}{\left|B_{2}\right|} \frac{\left|B_{1}\right|}{w\left(\bar{B}_{1}\right)} \leq C$ for all balls $B_{1}, B_{2}$ with $B_{1} \subset$ $B_{2}$. Thus, by Hölder' inequality and the reverse of Hölder' inequality for $w \in A_{1}$ and some $p>1$ with $1 / p+1 / p^{\prime}=1$, we obtain

$$
\begin{aligned}
J J \leq & C \sum_{|\alpha|=m}\left\|D^{\alpha} A\right\|_{B M O} \sum_{k=1}^{\infty} 2^{-k}\left(\frac{|B|}{w(B)} \frac{w\left(2^{k+1} B\right)}{\left|2^{k+1} B\right|}\right) \\
& +C \sum_{|\alpha|=m} \sum_{k=1}^{\infty} 2^{-k} \frac{|B|}{w(B)}\left(\frac{1}{\left|2^{k+1} B\right|} \int_{2^{k+1} B} w(x)^{p} d x\right)^{1 / p} \\
& \times\left(\frac{1}{\left|2^{k+1} B\right|} \int_{2^{k+1} B}\left|D^{\alpha} \tilde{A}(x)\right|^{p^{\prime}} d x\right)^{1 / p^{\prime}}
\end{aligned}
$$




$$
\begin{aligned}
& \leq C \sum_{|\alpha|=m}\left\|D^{\alpha} A\right\|_{B M O} \sum_{k=1}^{\infty} k 2^{-k}\left(\frac{w\left(2^{k+1} B\right)}{\left|2^{k+1} B\right|} \frac{|B|}{w(B)}\right) \\
& \leq C
\end{aligned}
$$

which together with the estimate for $J$ yields the desired result. This finishes the proof of Theorem 2 .

Proof of Theorem 3. By the equality

$$
R_{m+1}(A ; x, y)=Q_{m+1}(A ; x, y)+\sum_{|\alpha|=m} \frac{1}{\alpha !}(x-y)^{\alpha}\left(D^{\alpha} A(x)-D^{\alpha} A(y)\right)
$$

and the similarity to the proof of Lemma 2, we get

$$
\mu_{S}^{A}(f)(x) \leq \tilde{\mu}_{S}^{A}(f)(x)+C \sum_{|\alpha|=m} \int_{R^{n}} \frac{\left|D^{\alpha} A(x)-D^{\alpha} A(y)\right|}{|x-y|^{n}}|f(y)| d y .
$$

By Theorem 1, 2 and [2], we obtain

$$
\begin{aligned}
& w\left(\left\{x \in R^{n}: \mu_{S}^{A}(f)(x)>\lambda\right\}\right) \\
\leq & w\left(\left\{x \in R^{n}: \tilde{\mu}_{S}^{A}(f)(x)>\lambda / 2\right\}\right) \\
& +w\left(\left\{x \in R^{n}: \sum_{|\alpha|=m} \int_{R^{n}} \frac{\left|D^{\alpha} A(x)-D^{\alpha} A(y)\right|}{|x-y|^{n}}|f(y)| d y>C \lambda\right\}\right) \\
\leq & C\|f\|_{H^{1}(w)} / \lambda .
\end{aligned}
$$

This completes the proof of Theorem 3.

Proof of TheOREM 4. (i). It suffices to show that there exists a constant $C>0$ such that for every $H^{1}(w)$-atom $a$ with $\operatorname{supp} a \subset Q=$ $Q\left(x_{0}, d\right)$, there is

$$
\left\|\mu_{S}^{A}(a)\right\|_{L^{1}(w)} \leq C .
$$

Let $\tilde{A}(x)=A(x)-\sum_{|\alpha|=m} \frac{1}{\alpha !}\left(D^{\alpha} A\right)_{\tilde{Q}} x^{\alpha}$. We write, by the vanishing moment of $a$ and for $u \in 3 Q \backslash 2 Q$,

$$
\begin{aligned}
& F_{t}^{A}(a)(x, y) \\
=\quad & \chi_{4 Q}(x) F_{t}^{A}(a)(x, y)
\end{aligned}
$$




$$
\begin{aligned}
& +\chi_{(4 Q)^{c}}(x) \int_{R^{n}}\left[\frac{R_{m}(\tilde{A} ; x, z)}{|x-z|^{m}} \frac{\Omega(y-z)}{|y-z|^{n-1}} \chi_{\Gamma(y)}(z, t)\right. \\
& \left.-\frac{R_{m}(\tilde{A} ; x, u)}{|x-u|^{m}} \frac{\Omega(y-u)}{|y-u|^{n-1}} \chi_{\Gamma(y)}(u, t)\right] a(z) d z \\
& -\chi_{(4 Q)^{c}}(x) \sum_{|\alpha|=m} \frac{1}{\alpha !} \int_{R^{n}}\left[\frac{(x-z)^{\alpha}}{|x-z|^{m}} \frac{\Omega(y-z)}{|y-z|^{n-1}} \chi_{\Gamma(y)}(z, t)\right. \\
& \left.-\frac{(x-u)^{\alpha}}{|x-u|^{m}} \frac{\Omega(y-u)}{|y-u|^{n-1}} \chi_{\Gamma(y)}(u, t)\right] D^{\alpha} \tilde{A}(z) a(z) d z-\chi_{(4 Q)^{c}}(x) \\
& \times \sum_{|\alpha|=m} \frac{1}{\alpha !} \int_{R^{n}} \frac{(x-u)^{\alpha}}{|x-u|^{m}} \frac{\Omega(y-u)}{|y-u|^{n-1}} \chi_{\Gamma(y)}(u, t) D^{\alpha} \tilde{A}(z) a(z) d z,
\end{aligned}
$$

then

$$
\begin{aligned}
& \mu_{S}^{A}(a)(x) \\
= & \left\|\chi_{\Gamma(x)} F_{t}^{A}(a)(x, y)\right\| \\
\leq & \chi_{4 Q}(x)\left\|\chi_{\Gamma(x)} F_{t}^{A}(a)(x, y)\right\| \\
& +\chi_{(4 Q)^{c}}(x) \| \chi_{\Gamma(x)} \int_{R^{n}}\left[\frac{R_{m}(\tilde{A} ; x, z)}{|x-z|^{m}} \frac{\Omega(y-z) \chi_{\Gamma(y)}(z, t)}{|y-z|^{n-1}}\right. \\
& \left.-\frac{R_{m}(\tilde{A} ; x, u)}{|x-u|^{m}} \frac{\Omega(y-u) \chi_{\Gamma(y)}(u, t)}{|y-u|^{n-1}}\right] a(z) d z \| \\
& +\chi_{(4 Q)^{c}}(x) \| \sum_{|\alpha|=m} \frac{\chi_{\Gamma(x)}}{\alpha !} \int_{R^{n}}\left[\frac{(x-z)^{\alpha}}{|x-z|^{m}} \frac{\Omega(y-z) \chi_{\Gamma(y)}(z, t)}{|y-z|^{n-1}}\right. \\
& \left.-\frac{(x-u)^{\alpha}}{|x-u|^{m}} \frac{\Omega(y-u) \chi_{\Gamma(y)}(u, t)}{|y-u|^{n-1}}\right] D^{\alpha} \tilde{A}(z) a(z) d z \| \\
& +\chi_{(4 Q)^{c}}(x) \| \chi_{\Gamma(x)} \sum_{|\alpha|=m} \frac{1}{\alpha !} \int_{R^{n}} \frac{(x-u)^{\alpha}}{|x-u|^{m}} \frac{\Omega(y-u)}{|y-u|^{n-1}} \chi_{\Gamma(y)}(u, t) \\
& \times D^{\alpha} \tilde{A}(z) a(z) d y \| \\
= & K_{1}(x)+K_{2}(x, u)+K_{3}(x, u)+K_{4}(x, u) .
\end{aligned}
$$


By the $L^{p}(w)$-boundedness of $\mu_{S}^{A}$, we get

$$
\int_{R^{n}} K_{1}(x) w(x) d x=\int_{4 Q} \mu_{S}^{A}(a)(x) w(x) d x \leq C\|a\|_{L^{\infty}(w)} w(Q) \leq C
$$

For $K_{2}(x, u)$, we write

$$
\begin{aligned}
& \frac{R_{m}(\tilde{A} ; x, z)}{|x-z|^{m}} \frac{\Omega(y-z)}{|y-z|^{n-1}} \chi_{\Gamma(y)}(z, t) \\
& -\frac{R_{m}(\tilde{A} ; x, u)}{|x-u|^{m}} \frac{\Omega(y-u)}{|y-u|^{n-1}} \chi_{\Gamma(y)}(u, t) \\
= & \left(\chi_{\Gamma(y)}(z, t)-\chi_{\Gamma(y)}(u, t)\right) \frac{\Omega(y-z) R_{m}(\tilde{A} ; x, z)}{|y-z|^{n-1}|x-z|^{m}} \\
+ & {\left[\frac{\Omega(y-z)}{|y-z|^{n-1}}-\frac{\Omega(y-u)}{|y-u|^{n-1}}\right] \frac{R_{m}(\tilde{A} ; x, z)}{|x-z|^{m}} \chi_{\Gamma(y)}(u, t) } \\
+ & \frac{\Omega(y-u) \chi_{\Gamma(y)}(u, t)}{|y-u|^{n-1}}\left(\frac{R_{m}(\tilde{A} ; x, z)}{|x-z|^{m}}-\frac{R_{m}(\tilde{A} ; x, u)}{|x-u|^{m}}\right) .
\end{aligned}
$$

By the following inequality (see [18]):

$$
\left|\frac{\Omega(y-z)}{|y-z|^{n-1}}-\frac{\Omega(y-u)}{|y-u|^{n-1}}\right| \leq C\left(\frac{|z-u|}{|y-z|^{n}}+\frac{|z-u|^{\gamma}}{|y-z|^{n-1+\gamma}}\right)
$$

and note that

$$
\begin{aligned}
& || \chi_{\Gamma(x)} \int_{R^{n}}\left(\chi_{\Gamma(y)}(z, t)-\chi_{\Gamma(y)}(u, t)\right) \frac{\Omega(y-z) R_{m}(\tilde{A} ; x, z)}{|y-z|^{n-1}|x-z|^{m}} a(z) d z \| \\
\leq & C \int_{R^{n}} \frac{|a(z)|\left|R_{m}(\tilde{A} ; x, z)\right|}{|x-z|^{m}} \\
& \times\left(\iint_{R_{+}^{n+1}} \frac{\chi_{\Gamma(x)}(y, t)\left|\chi_{\Gamma(y)}(z, t)-\chi_{\Gamma(y)}(u, t)\right|^{2}}{|y-z|^{2 n-2}} \frac{d y d t}{t^{n+3}}\right)^{1 / 2} d z \\
\leq & C \int_{R^{n}} \frac{|a(z)|\left|R_{m}(\tilde{A} ; x, z)\right|}{|x-z|^{m}} \\
\times & \left|\iint_{\Gamma(x), \Gamma(z)} \frac{t^{-n-3} d y d t}{|y-z|^{2 n-2}}-\iint_{\Gamma(x), \Gamma(u)} \frac{t^{-n-3} d y d t}{|y-z|^{2 n-2}}\right|^{1 / 2} d z
\end{aligned}
$$




$$
\begin{aligned}
\leq & C \int_{R^{n}} \frac{|a(z)|\left|R_{m}(\tilde{A} ; x, z)\right|}{|x-z|^{m}}\left(\iint_{|y| \leq t,|x+y-z| \leq t}\left|\frac{1}{|x+y-z|^{2 n-2}}\right| \frac{1}{\mid y d t}\right)^{1 / 2} d z \\
& -\frac{1}{|x+y-u|^{2 n-2}} \mid \frac{|a(z)|\left|R_{m}(\tilde{A} ; x, z)\right|}{|x-z|^{m}} \\
\leq & \left.C \int_{R^{n}} \frac{|u-z| t^{-n-3} d y d t}{|x+y-z|^{2 n-1}}\right)^{1 / 2} d z \\
& \times\left(\iint_{|y| \leq t,|x+y-z| \leq t} \frac{|u-z|^{1 / 2}}{|x+z|^{n+1 / 2}} d z,\right. \\
\leq & C \int_{R^{n}} \frac{|a(z)|\left|R_{m}(\tilde{A} ; x, z)\right|}{|x-z|^{m}} \frac{\mid u-m}{|x-z|^{m}}
\end{aligned}
$$

by the similarity of the proof of Theorem 1 , we obtain

$$
\begin{aligned}
& \int_{R^{n}} K_{2}(x, u) w(x) d x \\
\leq & C \sum_{|\alpha|=m}\left\|D^{\alpha} A\right\|_{B M O} \sum_{k=2}^{\infty} \int_{2^{k+1} Q \backslash 2^{k} Q} \\
& \times \int_{Q} k\left(\frac{|u-z|}{|x-z|^{n+1}}+\frac{|u-z|^{1 / 2}}{|x-z|^{n+1 / 2}}+\frac{|u-z|^{\gamma}}{|x-z|^{n+\gamma}}\right)|a(z)| d z w(x) d x \\
\leq & C \sum_{|\alpha|=m}\left\|D^{\alpha} A\right\|_{B M O} \sum_{k=2}^{\infty} \int_{2^{k+1} Q \backslash 2^{k} Q} k \\
& \times\left(\frac{d}{\left(2^{k} d\right)^{n+1}}+\frac{d^{1 / 2}}{\left(2^{k} d\right)^{n+1 / 2}}+\frac{d^{\gamma}}{\left(2^{k} d\right)^{n+\gamma}}\right)\|a\|_{L}(w)|Q| w(x) d x \\
\leq & C \sum_{|\alpha|=m}\left\|D^{\alpha} A\right\|_{B M O} \sum_{k=2}^{\infty} k\left(2^{-k}+2^{-k / 2}+2^{-\gamma k}\right) \frac{w\left(2^{k+1} Q\right)}{\left|2^{k+1} Q\right|} \frac{|Q|}{w(Q)} \\
\leq & C \sum_{|\alpha|=m}\left\|D^{\alpha} A\right\|_{B M O} \sum_{k=2}^{\infty} k\left(2^{-k}+2^{-k / 2}+2^{-\gamma k}\right) \leq C ;
\end{aligned}
$$

Similarly, we get

$$
\int_{R^{n}} K_{3}(x, u) w(x) d x
$$


Weighted continuity of multilinear Marcinkiewicz operators

$$
\begin{aligned}
\leq & C \sum_{|\alpha|=m} \sum_{k=2}^{\infty} \int_{2^{k+1} Q \backslash 2^{k} Q} \int_{Q}\left(\frac{|u-z|}{|x-z|^{n+1}}+\frac{|u-z|^{1 / 2}}{|x-z|^{n+1 / 2}}\right. \\
& \left.+\frac{|u-z|^{\gamma}}{|x-z|^{n+\gamma}}\right)\left|D^{\alpha} \tilde{A}(z) \| a(z)\right| d z w(x) d x \\
\leq & C \sum_{|\alpha|=m} \sum_{k=2}^{\infty}\left(\frac{d}{\left(2^{k} d\right)^{n+1}}+\frac{d^{1 / 2}}{\left(2^{k} d\right)^{n+1 / 2}}+\frac{d^{\gamma}}{\left(2^{k} d\right)^{n+\gamma}}\right) \\
& \times\left(\frac{1}{|Q|} \int_{Q}\left|D^{\alpha} \tilde{A}(y)\right| d y\right)\|a\|_{L^{\infty}(w)}|Q| w\left(2^{k+1} Q\right) \\
\leq & C .
\end{aligned}
$$

Thus, by using the condition of $K_{4}(x, u)$, we obtain

$$
\int_{R^{n}} \mu_{S}^{A}(a)(x) w(x) d x \leq C .
$$

(ii). For any cube $Q=Q\left(x_{0}, d\right)$, let

$$
\tilde{A}(x)=A(x)-\sum_{|\alpha|=m} \frac{1}{\alpha !}\left(D^{\alpha} A\right)_{\tilde{Q}} x^{\alpha} .
$$

We write, for $f=f \chi_{4 Q}+f \chi_{(4 Q)^{c}}=f_{1}+f_{2}$ and $u \in 3 Q \backslash 2 Q$,

$$
\begin{aligned}
& \tilde{F}_{t}^{A}(f)(x, y) \\
= & \tilde{F}_{t}^{A}\left(f_{1}\right)(x, y)+\int_{|y-z| \leq t} \frac{R_{m}(\tilde{A} ; x, z)}{|x-z|^{m}} \frac{\Omega(y-z)}{|y-z|^{n-1}} f_{2}(z) d z \\
& -\sum_{|\alpha|=m} \frac{1}{\alpha !}\left(D^{\alpha} A(x)-\left(D^{\alpha} A\right)_{Q}\right) \\
& \times \int_{|y-z| \leq t}\left[\frac{(x-z)^{\alpha} \Omega(y-z)}{|x-z|^{m}|y-z|^{n-1}}-\frac{(u-z)^{\alpha} \Omega(y-z)}{|u-z|^{m}|y-z|^{n-1}}\right] f_{2}(z) d z \\
& -\sum_{|\alpha|=m} \frac{1}{\alpha !}\left(D^{\alpha} A(x)-\left(D^{\alpha} A\right)_{Q}\right) \\
& \times \int_{|y-z| \leq t} \frac{(u-z)^{\alpha}}{|u-z|^{m}} \frac{\Omega(y-z)}{|y-z|^{n-1}} f_{2}(z) d z,
\end{aligned}
$$


then

$$
\begin{aligned}
& \left|\tilde{\mu}_{S}^{A}(f)(x)-\mu_{S}\left(\frac{R_{m}\left(\tilde{A} ; x_{0}, \cdot\right)}{\left|x_{0}-\cdot\right|^{m}} f_{2}\right)\left(x_{0}\right)\right| \\
& =\|\| \chi_{\Gamma(x)} \tilde{F}_{t}^{A}(f)(x, y)\|-\| \chi_{\Gamma\left(x_{0}\right)} F_{t}\left(\frac{R_{m}\left(\tilde{A} ; x_{0}, \cdot\right)}{\left|x_{0}-\cdot\right|^{m}} f_{2}\right)(y)\|\| \\
& \leq\left\|\chi_{\Gamma(x)} \tilde{F}_{t}^{A}(f)(x, y)-\chi_{\Gamma\left(x_{0}\right)} F_{t}\left(\frac{R_{m}\left(\tilde{A} ; x_{0}, \cdot\right)}{\left|x_{0}-\right|^{m}} f_{2}\right)(y)\right\| \\
& \leq\left\|\chi_{\Gamma(x)}(y, t) \tilde{F}_{t}^{A}\left(f_{1}\right)(x, y)\right\| \\
& +\| \chi_{\Gamma(x)}(y, t) \int_{|y-z| \leq t}\left[\frac{R_{m}(\tilde{A} ; x, z) \Omega(y-z)}{|x-z|^{m}|y-z|^{n-1}}\right. \\
& \left.-\chi_{\Gamma\left(x_{0}\right)}(y, t) \int_{|y-z| \leq t} \frac{R_{m}\left(\tilde{A} ; x_{0}, z\right) \Omega(y-z)}{\left|x_{0}-z\right|^{m}|y-z|^{n-1}}\right] f_{2}(z) d z|| \\
& +\| \chi_{\Gamma(x)}(y, t) \sum_{|\alpha|=m} \frac{1}{\alpha !}\left(D^{\alpha} A(x)\right. \\
& \left.-\left(D^{\alpha} A\right)_{Q}\right) \int_{|y-z| \leq t}\left[\frac{\Omega(y-z)(x-z)^{\alpha}}{|y-z|^{n-1}|x-z|^{m}}\right. \\
& \left.-\frac{\Omega(y-z)(u-z)^{\alpha}}{|y-z|^{n-1}|u-z|^{m}}\right] f_{2}(z) d z|| \\
& +\| \chi_{\Gamma(x)}(y, t) \sum_{|\alpha|=m} \frac{1}{\alpha !}\left(D^{\alpha} A(x)\right. \\
& \left.-\left(D^{\alpha} A\right)_{Q}\right) \int_{|y-z| \leq t} \frac{\Omega(y-z)(u-z)^{\alpha}}{|y-z|^{n-1}|u-z|^{m}} f_{2}(z) d z \mid \\
& =L_{1}(x)+L_{2}(x)+L_{3}(x, u)+L_{4}(x, u) \text {. }
\end{aligned}
$$

By the $L^{p}(w)$-boundedness of $\tilde{\mu}_{S}^{A}$, we get

$$
\frac{1}{w(Q)} \int_{Q} L_{1}(x) w(x) d x \leq C\|f\|_{L^{\infty}(w)}
$$


For $L_{2}(x)$, we write

$$
\begin{aligned}
& \chi_{\Gamma(x)}(y, t) \frac{R_{m}(\tilde{A} ; x, z) \Omega(y-z)}{|x-z|^{m}|y-z|^{n-1}} \\
& -\chi_{\Gamma\left(x_{0}\right)}(y, t) \frac{R_{m}\left(\tilde{A} ; x_{0}, z\right) \Omega(y-z)}{\left|x_{0}-z\right|^{m}|y-z|^{n-1}} \\
& =\quad \chi_{\Gamma(x)}(y, t)\left[\frac{R_{m}(\tilde{A} ; x, z)}{|x-z|^{m}}-\frac{R_{m}\left(\tilde{A} ; x_{0}, z\right)}{\left|x_{0}-z\right|^{m}}\right] \frac{\Omega(y-z)}{|y-z|^{n-1}} \\
& +\left(\chi_{\Gamma(x)}(y, t)-\chi_{\Gamma\left(x_{0}\right)}(y, t)\right) \frac{R_{m}\left(\tilde{A} ; x_{0}, z\right)}{\left|x_{0}-z\right|^{m}} \frac{\Omega(y-z)}{|y-z|^{n-1}},
\end{aligned}
$$

then, by similarity to the proof of Lemma 2 and $K_{2}(x, u)$, we obtain

$$
\begin{aligned}
& \frac{1}{w(Q)} \int_{Q} L_{2}(x) w(x) d x \\
\leq & C \sum_{|\alpha|=m}\left\|D^{\alpha} A\right\|_{B M O} \sum_{k=2}^{\infty} \int_{2^{k+1} Q \backslash 2^{k} Q} k \\
& \times\left(\frac{\left|x-x_{0}\right|}{|x-y|^{n+1}}+\frac{\left|x-x_{0}\right|^{1 / 2}}{|x-y|^{n+1 / 2}}+\frac{\left|x-x_{0}\right|^{\gamma}}{|x-y|^{n+\gamma}}\right)|f(y)| d y \\
\leq & C\|f\|_{L^{\infty}(w)}
\end{aligned}
$$

Similarly, we get

$$
\frac{1}{w(Q)} \int_{Q} L_{3}(x, u) w(x) d x \leq C\|f\|_{L^{\infty}(w)} .
$$

Thus, by using the condition of $L_{4}(x, u)$, we obtain

$$
\begin{aligned}
& \frac{1}{w(Q)} \int_{Q}\left|\tilde{\mu}_{S}^{A}(f)(x)-\mu_{S}\left(\frac{R_{m}\left(\tilde{A} ; x_{0}, \cdot\right)}{\left|x_{0}-\cdot\right|^{m}} f_{2}\right)\left(x_{0}\right)\right| w(x) d x \\
\leq & C\|f\|_{L^{\infty}(w)} .
\end{aligned}
$$

This completes the proof of Theorem 4.

ACKNOWLEDGEMENT. The author would like to express his gratitude to the referee for his comments and suggestions.

\section{References}

[1] Bui Huy Qui, Weighted hardy spaces, Math. Nachr. 103 (1981), 45-62.

[2] S. Chanillo, A note on commutators, Indiana Univ. Math. J. 31 (1982), 7-16. 
[3] W. Chen and G. Hu, Weak type $\left(H^{1}, L^{1}\right)$ estimate for multilinear singular integral operator, Adv. Math.(China) 30 (2001), 63-69.

[4] J. Cohen, $A$ sharp estimate for a multilinear singular integral on $R^{n}$, Indiana Univ. Math. J.30 (1981), 693-702.

[5] J. Cohen and J. Gosselin, On multilinear singular integral operators on $R^{n}$, Studia Math. 72 (1982), 199-223.

[6] — A BMO estimate for multilinear singular integral operators, Illinois J. Math. 30 (1986), 445-465.

[7] R. Coifman, R. Rochberg and G. Weiss, Factorization theorems for Hardy spaces in several variables, Ann. of Math. 103 (1976), 611-635.

[8] Y. Ding, A note on multilinear fractional integrals with rough kernel, Adv. Math. (China) 30 (2001), 238-246.

[9] Y. Ding and S. Z. Lu, Weighted boundedness for a class rough multilinear operators, Acta Math. Sinica 3 (2001), 517-526.

[10] Y. Ding, S. Z. Lu and Q. Xue, On Marcinkiewicz integral with homogeneous kernels, J. Math. Anal. Appl. 245 (2000), 471-488.

[11] J. Garcia-Cuerva and J. L. Rubio de Francia, Weighted norm inequalities and related topics, North-Holland Math. 16, Amsterdam, 1985.

[12] E. Harboure, C. Segovia and J. L. Torrea, Boundedness of commutators of fractional and singular integrals for the extreme values of $p$, Illinois J. Math. 41 (1997), 676-700.

[13] G. Hu and D. C. Yang, $A$ variant sharp estimate for multilinear singular integral operators, Studia Math. 141 (2000), 25-42.

[14] , Multilinear oscillatory singular integral operators on Hardy spaces, Chinese J. Contemp. Math. 18 (1997), 403-413.

[15] Liu Lanzhe, Boundedness for multilinear Marcinkiewicz Operators on certain Hardy Spaces, Inter. J. Math. Math. Sci. 2 (2003), 87-96.

[16] C. Perez, Endpoint estimate for commutators of singular integral operators, J. Funct. Anal. 128 (1995), 163-185.

[17] A. Torchinsky, The real variable methods in harmonic analysis, Pure and Applied Math. 123, Academic Press, New York, 1986.

[18] A. Torchinsky and S. Wang, A note on the Marcinkiewicz integral, Colloq. Math. 60/61 (1990), 235-243.

College of Mathematics and Computer Changsha University of Science and Technology

Changsha 410077, P. R. China

E-mail: lanzheliu@263.net 